\title{
SOBREVIVÊNCIA E CRESCIMENTO DE JUVENIS DO LINGUADO PARALICHTHYS ORBIGNYANUS CRIADOS EM DIFERENTES TEMPERATURAS
}

\author{
MARCELO HIDEO OKAMOTO \& LUÍS ANDRÉ SAMPAIO \\ Universidade Federal do Rio Grande - Instituto de Oceanografia, Laboratório de Piscicultura Estuarina e Marinha, CP 474, Rio Grande - RS - \\ Brasil. CEP: 96201-900 - mar_okamoto@yahoo.com.br; sampaio@mikrus.com.br
}

\section{RESUMO}

O linguado Paralichthys orbignyanus é um importante recurso pesqueiro que está despertando o interesse de sua produção em cativeiro. Este trabalho avaliou o efeito da temperatura sobre juvenis do linguado. Juvenis $(32,3 \pm 1,6 \mathrm{mg})$ foram mantidos nas temperaturas de $17,20,23$ e $26^{\circ} \mathrm{C}$, todas com duas repetições. A cada duas semanas, durante 56 dias, todos os peixes foram medidos e pesados. O tratamento estatístico dos resultados foi feito por Análise de Variância (uma via) seguida do Teste de Tukey, com $95 \%$ de significância. A sobrevivência foi de $100 \%$ em todos os tratamentos, indicando que logo após a metamorfose o linguado já é euritérmico. A taxa de crescimento específico diário foi de $5,25 \pm 0,06 \%\left(23^{\circ} \mathrm{C}\right), 4,87 \pm 0,23 \%$ $\left(26^{\circ} \mathrm{C}\right), 4,66 \pm 0,21 \%\left(20^{\circ} \mathrm{C}\right)$ e $4,07 \pm 0,14 \%\left(17^{\circ} \mathrm{C}\right)$, havendo diferença significativa apenas entre 23 e $17^{\circ} \mathrm{C}(\mathrm{P}<0,05)$. Foi observada diferença significativa apenas no peso final dos linguados criados a $23^{\circ} \mathrm{C}(608,8 \pm 76,3 \mathrm{mg})$ e $17^{\circ} \mathrm{C}(318,7 \pm 22,1$ $\mathrm{mg})(\mathrm{P}<0,05)$. De acordo com os resultados obtidos neste trabalho, sugere-se que o berçário de $P$. orbignyanus seja realizado entre 20 e $26^{\circ} \mathrm{C}$.

PALAVRAS CHAVE: Paralichthys orbignyanus, piscicultura, temperatura

\begin{abstract}
Survival and growth of juvenile Brazilian flounder Paralichthys orbignyanus reared at different temperatures The Brazilian flounder Paralichthys orbignyanus is an important fisheries resource and it is being considered for aquaculture. This study evaluated the effect of temperature on juvenile flounder. Fish $(32.3 \pm 1.6 \mathrm{mg})$ were reared at $17,20,23 \mathrm{and} 26^{\circ} \mathrm{C}$, with duplicate tanks for each temperature. Every two weeks, during 56 days, all fish were measured and weighed. Statistical treatment of results was done by One-Way ANOVA followed by the Tukey's Test, with $95 \%$ significance. Survival was $100 \%$ in all treatments, suggesting that Brazilian flounder is eurythermic soon after completing metamorphosis. The daily specific growth rate was $5.25 \pm 0.06 \%\left(23^{\circ} \mathrm{C}\right), 4.87 \pm 0.23 \%\left(26^{\circ} \mathrm{C}\right), 4.66 \pm 0.21 \%\left(20^{\circ} \mathrm{C}\right)$ and $4.07 \pm 0.14 \%\left(17^{\circ} \mathrm{C}\right)$ with significant difference only between 23 and $17^{\circ} \mathrm{C}(\mathrm{P}<0.05)$. Significant difference was observed only in the final weight of flounders reared at $23^{\circ} \mathrm{C}$ $(608.8 \pm 76.3 \mathrm{mg})$ and $17^{\circ} \mathrm{C}(318.7 \pm 22.1 \mathrm{mg})(\mathrm{P}<0.05)$. According to the results obtained in this study, it is suggested that juvenile $P$. orbignyanus should be reared in temperatures between 20 and $26^{\circ} \mathrm{C}$.
\end{abstract}

KEYWORDS: fish culture, Paralichthys orbignyanus, temperature

\section{INTRODUÇÃO}

O linguado Paralichthys orbignyanus (Valenciennes 1839) habita a plataforma continental do Oceano Atlântico Sul Ocidental, desde a costa até aproximadamente $20 \mathrm{~m}$ de profundidade. Sua distribuição se estende do estado do Rio de Janeiro (Brasil) até Mar del Plata (Argentina), sendo comum sua ocorrência associada a regiões estuarinas (Figueiredo \& Menezes 2000). Seu valor comercial é elevado e é um importante item da pesca artesanal e industrial na costa do Rio Grande do Sul. Alguns exemplares podem atingir mais de $100 \mathrm{~cm}$ de comprimento total e aproximadamente $10 \mathrm{~kg}$ (Bianchini et al. 2010).

Além do elevado valor comercial, o linguado tem capacidade de tolerar ampla faixa de $\mathrm{pH}$ (Wasielesky et al. 1997), de temperatura (Wasielesky et al. 1998) e de salinidade (Sampaio \& Bianchini 2002), bem como concentrações elevadas de compostos nitrogenados (Bianchini et al. 1996), o que o caracteriza como uma espécie promissora para piscicultura marinha.

A desova de linguado pode ser obtida de forma espontânea em cativeiro, pela manipulação do fotoperíodo e da temperatura ou pode ser induzida, com a aplicação de hormônios (Sampaio et al. 2008). Estudos realizados sobre a larvicultura do linguado permitiram a elaboração de um protocolo para a produção de juvenis de forma rotineira com boas taxas de sobrevivência (Sampaio et al. 2007; Sampaio et al. 2008).

A temperatura da água é um dos fatores ambientais mais importantes que afetam o crescimento de peixes (Brett \& Groves 1979). Para organismos ectotérmicos, como os peixes, existem faixas de temperatura em que o crescimento é favorecido. Em temperaturas inferiores a queda do metabolismo reduz o crescimento, enquanto que com - aquecimento acima da faixa de temperatura considerada ideal, também ocorre redução do crescimento (Baldisserotto 2009).

Este trabalho avaliou a sobrevivência e o crescimento de juvenis de $P$. orbignyanus criados, na fase de berçário, em diferentes temperaturas.

\section{MATERIAIS E MÉTODOS}

Linguados adultos, capturados com rede de arrasto na Praia do Cassino (Rio Grande - RS) 
durante sua época reprodutiva, foram levados para a Estação Marinha de Aquacultura - FURG, onde foram condicionados ao cativeiro. As fêmeas foram submetidas à biópsia para a determinação do estádio de desenvolvimento gonadal $e$, as que se apresentaram aptas à indução hormonal (oócitos com diâmetro aproximadamente de $400 \mu \mathrm{m}$ ), receberam uma única injeção intramuscular de extrato de pituitária de carpa na dose de $5 \mathrm{mg} / \mathrm{kg}$ (Sampaio et al. 2008). Ovócitos e sêmen foram coletados por meio de extrusão manual e os espermatozóides foram ativados com água do mar (salinidade 30) para a realização da fertilização artificial.

As larvas foram criadas durante 30 dias em um tanque de $1.000 \mathrm{~L}$ contendo água do mar de salinidade $30,2 \pm 0,1$ constantemente aerada, mantida a $23,4 \pm 0,3^{\circ} \mathrm{C}$ e sob um fotoperíodo de $24 \mathrm{~h}$ de luz (Sampaio et al. 2006, Sampaio et al. 2007). Durante o estádio larval, rotíferos e náuplios de Artemia foram oferecidos como alimento.

Ao final da metamorfose, os juvenis de linguado $(32,3 \pm 1,6 \mathrm{mg}$ e 14,8 $\pm 0,3 \mathrm{~mm})$ foram distribuídos aleatoriamente em oito tanques de $15 \mathrm{~L}$ (30 peixes por tanque). No momento da transferência, a temperatura e a salinidade da água eram iguais a $23^{\circ} \mathrm{C}$ e 30 , respectivamente. Posteriormente, as temperaturas foram ajustadas a uma taxa de $3^{\circ} \mathrm{C} \mathrm{h}^{-1}$ para 17,20 e $26^{\circ} \mathrm{C}$, todas com duas repetições.

$\mathrm{O}$ experimento foi realizado em laboratório climatizado a $17^{\circ} \mathrm{C}$. As demais temperaturas foram mantidas com aquecedores submersíveis de $300 \mathrm{~W}$ equipados com termostato. Um reservatório com água aquecida e outro com água fria foram mantidos para a renovação da água dos tanques. A água aquecida foi misturada com a água fria até a obtenção da temperatura desejada em um recipiente à parte e só então foi adicionada aos tanques para evitar que os peixes sofressem choque térmico.

Os peixes foram alimentados inicialmente com náuplios de Artemia até o $23^{\circ}$ dia de experimento. A partir de então teve início o desmame com ração comercial (INVE NRD). Os restos de alimento e fezes acumulados no fundo dos tanques foram retirados com um sifão. Cerca de $80 \%$ do volume d'água foi renovado uma vez ao dia, durante o período de alimentação com Artemia e duas vezes ao dia (08:00h e 17:00h) a partir do oferecimento de ração.

O experimento teve duração de 56 dias e a cada duas semanas todos os peixes foram anestesiados com benzocaína (50 ppm), medidos e pesados para o acompanhamento do crescimento. Com os dados obtidos foi calculada a taxa de crescimento específico diário: TCE $=[(\ln \mathrm{pf}-\ln \mathrm{pi}) / \mathrm{t}]$ x 100, onde pi e pf são os pesos (g) dos juvenis no início e ao final do experimento, respectivamente, e $t$ é o tempo de experimento em dias.

A temperatura e a concentração de oxigênio dissolvido ao longo do experimento foram 17,2 \pm $0,1^{\circ} \mathrm{C}$ e $6,94 \pm 0,10 \mathrm{mg} \mathrm{O}_{2} \mathrm{~L}^{-1} ; 20,0 \pm 0,1^{\circ} \mathrm{C}$ e $6,49 \pm$ $0,08 \mathrm{mg} \mathrm{O}_{2} \mathrm{~L}^{-1} ; 22,7 \pm 0,0^{\circ} \mathrm{C}$ e $6,06 \pm 0,06 \mathrm{mg} \mathrm{O}_{2} \mathrm{~L}^{-1}$; e $26,0 \pm 0,1^{\circ} \mathrm{C}$ e $5,69 \pm 0,08 \mathrm{mg} \mathrm{O}_{2} \mathrm{~L}^{-1}$. A salinidade permaneceu em $32,4 \pm 0,3 \mathrm{em}$ todos os tratamentos.

O tratamento estatístico dos resultados (média \pm erro padrão) foi feito por meio de Análise de Variância (uma via) ao nível de significância de 95\% e as diferenças foram identificadas com a aplicação do Teste de Tukey (Sokal \& Rohlf 1995). Estas análises foram realizadas com o programa Statistica 7.0.

\section{RESULTADOS}

A sobrevivência foi de $100 \%$ em todas as temperaturas testadas $(P>0,05)$. Logo na primeira biometria, os peixes mantidos a $23^{\circ} \mathrm{C}$ apresentaram peso significativamente superior aos demais $(P<0,05)$. Também houve diferença significativa $(P<0,05)$ entre o peso dos peixes mantidos a $26 \mathrm{e}$ $17^{\circ} \mathrm{C}$, porém ambos não diferiram do peso dos peixes mantidos a $20^{\circ} \mathrm{C}(P>0,05)$. Entretanto, esta diferença foi desaparecendo ao longo do tempo e ao final do experimento foi observada diferença significativa apenas entre os linguados criados a 23 e $17^{\circ} \mathrm{C}$ $(P<0,05)$ (Figura 1). 


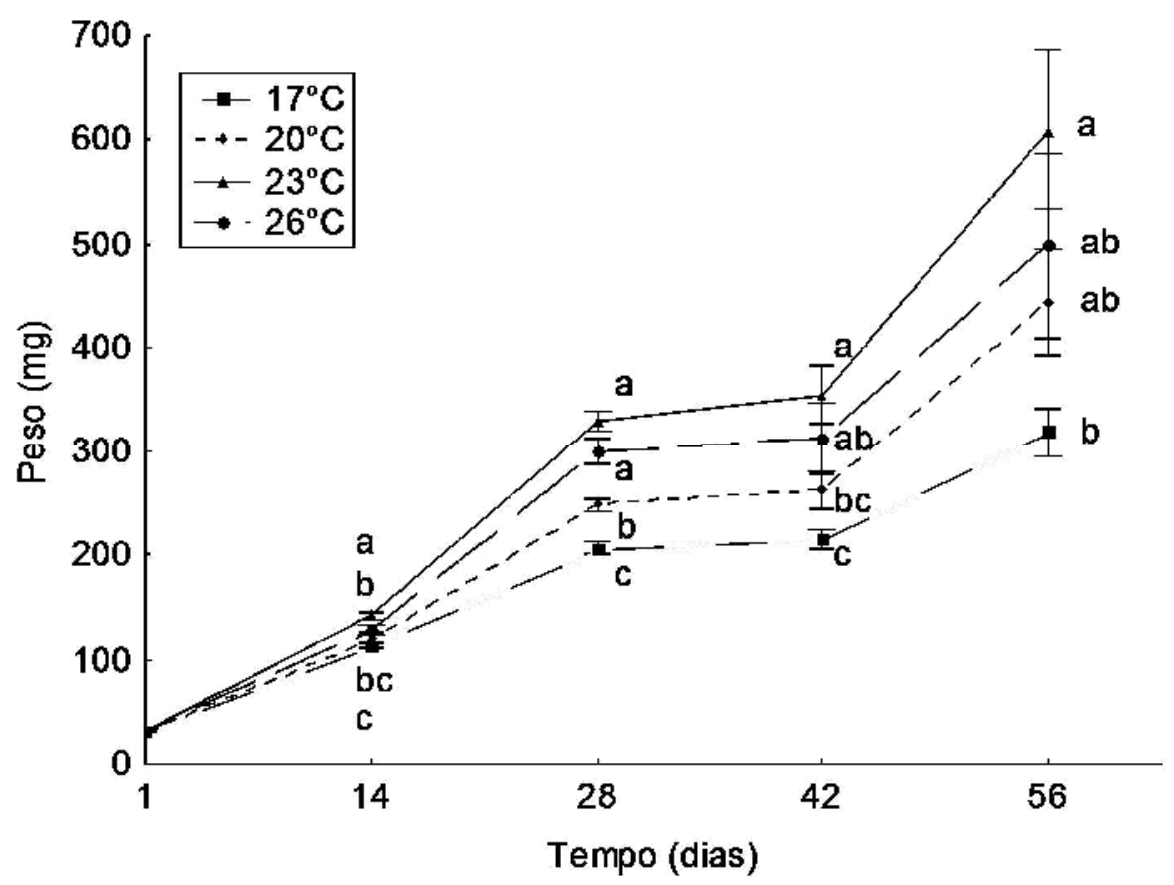

FIGURA 1 - Peso (média \pm EP) de juvenis do linguado Paralichthys orbignyanus criados em diferentes temperaturas. Letras diferentes indicam diferença significativa $(P<0,05)$ entre os tratamentos em cada intervalo de tempo.

Os juvenis mantidos em 17,20 e $26^{\circ} \mathrm{C}$ apresentaram taxas de crescimento específico diário acima de $4 \% \mathrm{dia}^{-1}$, enquanto que para aqueles mantidos a $23^{\circ} \mathrm{C}$ a taxa de crescimento específico foi superior a $5 \% \mathrm{dia}^{-1}$ (Figura 2), havendo diferença significativa apenas entre os tratamentos 17 e $23^{\circ} \mathrm{C}$ $(\mathrm{P}<0,05)$.

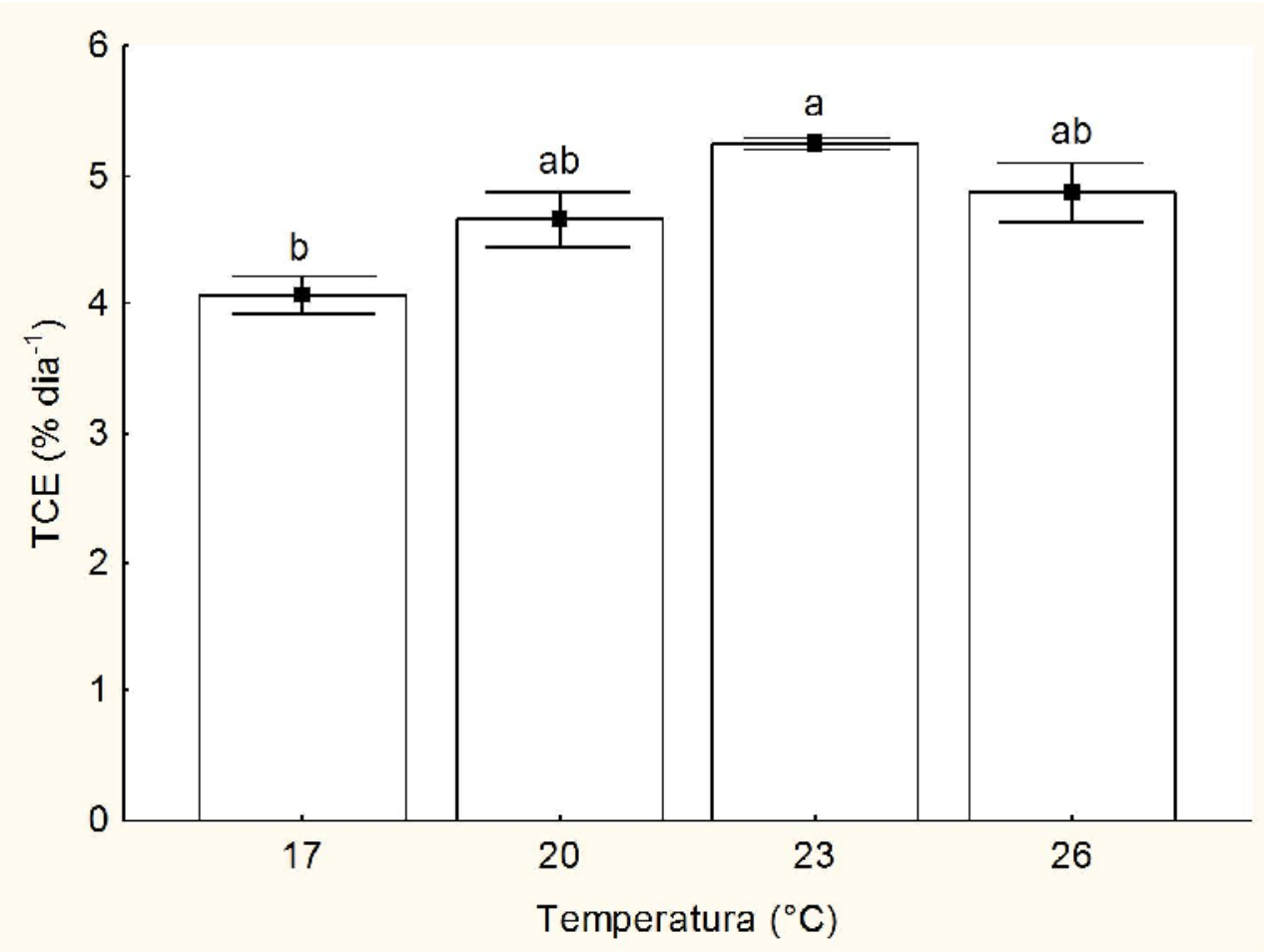

FIGURA 2 - Taxa de crescimento específico diário (TCE) (média \pm EP) de juvenis do linguado Paralichthys orbignyanus criados em diferentes temperaturas. Letras diferentes indicam diferença significativa entre os tratamentos $(P<0,05)$. 


\section{DISCUSSÃO}

Em ensaios de tolerância à temperatura, foi observado que juvenis de $P$. orbignyanus $(109 \pm 11 \mathrm{~g}$ ) são euritérmicos, pois sobrevivem entre 10 e $27^{\circ} \mathrm{C}$. Em temperaturas abaixo ou acima desta faixa, a digestão foi prejudicada, provocando regurgitação do alimento seguida posteriormente de morte (Wasielesky et al. 1998). No presente trabalho foi avaliada uma faixa de temperatura menor $\left(17-26^{\circ} \mathrm{C}\right)$. A sobrevivência total, aliada à procura por alimento em todas as temperaturas, mostram que logo após a metamorfose, o linguado já é euritérmico. Juvenis de tainha Mugil liza (870 $\pm 250 \mathrm{mg})$ e de peixe-rei Odontesthes argentinensis (161 $\pm 3 \mathrm{mg}$ ) também apresentaram elevada sobrevivência, quando criados entre $20-30^{\circ} \mathrm{C}$ e $20-26^{\circ} \mathrm{C}$, respectivamente (Okamoto et al. 2006; Streit et al. 2010). Estas duas espécies, assim como $P$. orbignyanus, habitam regiões costeiras e estuarinas e são interessantes para a criação em áreas como o Estuário da Lagoa dos Patos e região costeira adjacente, onde podem ocorrer variações de temperatura entre $9-28^{\circ} \mathrm{C}$ (Baumgarten \& Niencheski 1990).

A perda de diferença significativa do peso dos linguados criados nas diferentes temperaturas ao longo do tempo foi resultado do crescimento heterogêneo apresentado por esta espécie. Podemos observar na Figura 1 que entre $\circ 28^{\circ}$ e $\circ 42^{\circ}$ dias houve uma desaceleração no crescimento dos linguados em todos os tratamentos. Essa redução no crescimento pode estar associada ao processo de desmame, período em que os peixes tiveram que se adaptar à alimentação exclusivamente com ração. A partir do $42^{\circ}$ dia houve uma recuperação no crescimento dos linguados. $O$ crescimento heterogêneo dos linguados também resultou em desvios padrões amplos da TCE, ocasionando diferença significativa apenas entre os tratamentos 17 e $23^{\circ} \mathrm{C}$.

Juvenis do linguado Paralichthys lethostigma $\left(280 \pm 20 \mathrm{mg}\right.$ ) criados em 23 e $28^{\circ} \mathrm{C}$ não apresentaram diferença significativa na TCE, porém 0 peso médio final foi maior naqueles criados na menor temperatura (Luckenbach et al. 2007). Esta diferença de tamanho foi explicada pela maior expressão do RNAm do fator de crescimento tipo insulina I (IGF-I) no músculo, além da maior quantidade de IGF-I plasmático no sangue dos peixes criados a $23^{\circ} \mathrm{C}$. $\mathrm{O}$ IGF-I é o principal mediador da ação fisiológica do hormônio do crescimento (Moriyama et al. 2000) e trabalhos futuros analisando a expressão destes genes em relação à temperatura devem ser realizados com juvenis de $P$. orbignyanus.

A temperatura possui forte influência na determinação do sexo em algumas espécies de peixes, podendo provocar alterações na proporção entre machos e fêmeas (Devlin \& Nagahama 2002). Já foi reportado que a determinação do sexo do linguado japonês Paralichthys olivaceus é dependente da temperatura. Quando juvenis desta espécie, ainda com as gônadas indiferenciadas, são mantidos em temperatura baixa $\left(15^{\circ} \mathrm{C}\right)$ ou alta $\left(25^{\circ} \mathrm{C}\right)$, ocorre um maior percentual de machos, enquanto que em temperaturas intermediárias a proporção entre machos e fêmeas fica próxima de 1:1 (Yamamoto 1999). Luckenbach et al. (2003) observaram que a determinação do sexo de $P$. lethostigma também segue este padrão. Como a temperatura tem influência na determinação do sexo destas duas espécies de Paralichthys, é importante investigar se o mesmo ocorre em $P$. orbignyanus.

O crescimento heterogêneo observado neste experimento é um fator indesejável na piscicultura (Ostrensky \& Boeger 1998). Esta heterogeneidade pode estar parcialmente ligada ao sexo, já que estudos em populações selvagens demonstram que fêmeas de $P$. orbignyanus crescem mais rápido que machos (Cazorla 2005). Por tanto, a produção monossexo de fêmeas poderia ser um meio de corrigir este problema e otimizar a produção de linguado.

Pelos resultados obtidos neste trabalho, sugere-se que a criação de juvenis de $P$. orbignyanus seja realizada entre 20 e $26^{\circ} \mathrm{C}$.

\section{AGRADECIMENTOS}

Os autores agradecem a CAPES pela Bolsa de Doutorado para M.H. Okamoto e ao CNPq pela Bolsa de Produtividade em Pesquisa para L.A. Sampaio (Processo 308013/2009-3). Esse trabalho foi parcialmente financiado pela FAPERGS (Processo 9505355) e CNPq (Processo 480397/2010-4 e 556426/2009-6).

\section{REFERÊNCIAS}

BALDISSEROTTO, B. 2009. Fisiologia de peixes aplicada à piscicultura. Santa Maria, Editora UFSM. 350p. 
BAUMGARTEN, MGZ \& LF NIENCHESKI. 1990. Estuário da Laguna dos Patos: variações de alguns parâmetros físicoquímicos da água e metais associados ao material em suspensão. Cienc. Cult., 42(5/6): 390-396.

BIANCHINI, A, RB ROBALDO \& LA SAMPAIO. 2010. Cultivo do linguado (Paralichthys orbignyanus). In: BALDISSEROTO, B \& LC GOMES (eds.). Espécies nativas para piscicultura no Brasil. Editora UFSM, Brasil, Chap. 22: 559-587.

BIANCHINI, A, W WASIELESKY \& KC MIRANDA. 1996. Toxicity of nitrogenous compounds to juveniles of flatfish Paralichthys orbignyanus. Bull. Environ. Contam. Toxicol., 56: 453-459.

BRETT, JR \& TDD GROVES. 1979. Physiological energetics. In: HOAR, WS, DJ RANDALL \& JR BRETT (eds.). Bioenergetics and growth. Fish physiology, volume VIII. Academic Press, Inglaterra, Chap. 6: 280-352.

CAZORLA, AL. 2005. On the age and growth of flounder Paralichthys orbignyanus (Jenins, 1842) in Bahía Blanca Estuary, Argentina. Hidrobiologia, 537: 81-87.

DEVLIN, RH \& Y NAGAHAMA. 2002. Sex determination and sex differentiation in fish: an overview of genetic, physiological and environmental influences. Aquaculture, 208: 191-364.

FIGUEIREDO, JL \& NA MENEZES. 2000. Manual de peixes marinhos do sudeste do Brasil. São Paulo, Museu de Zoologia / USP. $116 \mathrm{p}$.

LUCKENBACH, JA, J GODWIN, HV DANIELS, RJ BOSRKI. 2003. Gonadal differentiation and effects of temperature on sex determination in southern flounder (Paralichthys lethostigma). Aquaculture, 216: 315-327.

LUCKENBACH, JA, R MURASHIGE, HV DANIELS, J GODWIN \& RJ BORSKI. 2007. Temperature affects insulin-like growth factor I and growth of juvenile southern flounder, Paralichthys lethostigma. Comp. Biochem. Phys. A, 146: 95-104.

MORIYAMA, S, FG AYSON \& H KAWAUCHI. 2000. Growth regulation by insulin-like growth factor-I in fish. Biosci. Biotechnol. Biochem. 64: 1553-1562.

OKAMOTO, MH, LA SAMPAIO \& AP MAÇADA. 2006. Efeito da temperatura sobre o crescimento e a sobrevivência de juvenis da tainha Mugil platanus Günther, 1880. Atlântica. 28(1): 61-66. OSTRENSKY, A \& W BOEGER. 1998. Piscicultura: fundamentos e técnicas de manejo. Guaíba, Editora Agropecuária. 211p.

SAMPAIO, LA \& A BIANCHINI. 2002. Salinity effects on osmoregulation and growth of the euryhaline flounder Paralichthys orbignyanus. J. Exp. Mar. Biol. Ecol., 269: 187-196.

SAMPAIO, LA, RB ROBALDO, MH OKAMOTO, LR LOUZADA, LS FREITAS, RV RODRIGUES \& A BIANCHINI. 2006. Reprodução e larvicultura do linguado Paralichthys orbignyanus: revisão do conhecimento atual. In: CYRINO, JEP \& EC URBINATI (eds.). Aquaciência 2004 - Tópicos especiais em biologia aquática e aqüicultura. Costa Ramos Campinas Ltda-ME, Brasil, Chap. 9: 117-124.

SAMPAIO, LA, LS FREITAS, M OKAMOTO, LR LOUZADA, RV RODRIGUES, \& RB ROBALDO. 2007. Effects of salinity on Brazilian flounder Paralichthys orbignyanus from fertilization to juvenile settlement. Aquaculture, 262: 340-346.

SAMPAIO, LA, RB ROBALDO \& A BIANCHINI. 2008. Hormone induced ovulation, natural spawning and larviculture of Brazilian flounder Paralichthys orbignyanus (Valenciennes, 1839). Aquac. Res., 39: 712-717.

SOKAL, RR \& FJ ROHLF. 1995. Biometry. Nova lorque, W. H. Freeman and Company. 887p.

STREIT, DP, MB TESSER, D BURKERT, CC SANCHEZ \& LA SAMPAIO, 2010. Survival and growth of juvenile marine pejerrey, Odontesthes argentinensis, reared at different temperatures. J. World Aquac. Soc., 41(6): 931-935.

WASIELESKY, W, A BIANCHINI, MHS SANTOS \& LH POERSCH. 1997. Tolerance of juvenile flatfish Paralichthys orbignyanus to acid stress. J. World Aquac. Soc., 28: 202-204.

WASIELESKY, W, A BIANCHINI \& K MIRANDA. 1998. Tolerancia a la temperatura de juveniles de lenguado Paralichthys orbignyanus. Frente Marítimo, 17 (A): 43-48.

YAMAMOTO, E. 1999. Studies on sex-manipulation and production of cloned populations in hirame, Paralichthys olivaceus (Temminck et Schlegel). Aquaculture, 173: 235-246.

Submetido $-21 / 10 / 2011$

Aceito - 15/02/2012 
